\title{
Coblation Intracapsular Tonsillectomy and Coblation Complete Tonsillectomy for Obstructive Sleep Apnea
}

\author{
Itzhak Braverman ${ }^{1}$, Alex Nemirovsky², Adi Klein'2, Miriam Sarid ${ }^{3}$, Galit Avior ${ }^{1}$ \\ ${ }^{1}$ Unit of Otolaryngology-Head \& Neck Surgery, Hadera, Israel \\ ${ }^{2}$ Pediatric Department Hillel Yaffe Medical Center, Hadera and The Faculty of Medicine, The Technion, Haifa, Israel \\ ${ }^{3}$ Western Galilee College, Acco, Israel \\ Email: braverman@hy.health.gov.il
}

Received 20 July 2015; accepted 8 September 2015; published 11 September 2015

Copyright (C) 2015 by authors and Scientific Research Publishing Inc.

This work is licensed under the Creative Commons Attribution International License (CC BY). http://creativecommons.org/licenses/by/4.0/

(c) (i) Open Access

\begin{abstract}
Objective: Total tonsillectomy and intracapsular tonsillectomy are common procedures for the treatment of obstructive sleep apnea (OSA) in children. The objective of this study was to compare the effectiveness of coblation intracapsular tonsillectomy (ICT) and coblation complete tonsillectomy (CT) as treatments for OSA. Study design: A retrospective study of all the children ages 2 18 years with OSA who underwent coblation intracapsular tonsillectomy (ICT) or coblation complete tonsillectomy (CT) from January 2007 to August 2010 by the same surgeons at one institution. Methods: Data were retrieved from children's charts and from telephone interviews with children's parents, regarding pre and postoperative OSA-18 scores, postoperative pain, postoperative complications, use of analgesic drugs, and time to return to a solid food diet. Results: All 43 children who underwent ICT and 37 children who underwent CT suffered from OSA before surgery, and none did postoperatively. There were no minor complications in the ICT group, compared to $13.5 \%$ in the CT group $(p=0.01)$. According to parental report, $72 \%$ and $21 \%$ suffered a low level of postoperative pain, and $9 \%$ and $33 \%$ severe pain in the ICT and CT groups, respectively. For these respective groups, $49 \%$ and $73 \%$ needed analgesic drugs $(p<0.05)$; and $65 \%$ and $35 \%$ ate solid food during the first 3 days post surgery. Conclusions: Both ICT and CT were safe, with few complications; however recovery was faster in the ICT group, as demonstrated by less pain, and more rapid return to a solid food diet.
\end{abstract}

\section{Keywords}

Obstructive Sleep Apnea, Tonsillectomy, Coblation, Intracapsular Tonsillectomy, Pain, Adenotonsillectomy, Tonsillotomy

\footnotetext{
${ }^{*}$ Corresponding author.
}

How to cite this paper: Braverman, I., Nemirovsky, A., Klein, A., Sarid, M. and Avior, G. (2015) Coblation Intracapsular Tonsillectomy and CoblationComplete Tonsillectomy for Obstructive Sleep Apnea. International Journal of Otolaryngology and Head \& Neck Surgery, 4, 350-355. http://dx.doi.org/10.4236/ijohns.2015.45059 


\section{Introduction}

Obstructive sleep apnea (OSA) in children is characterized by a decrease or complete halt in airflow despite an ongoing effort to breath, and by upper airway collapse that disrupts normal respiratory gas exchange or causes sleep fragmentation. A systematic review concluded that $4 \%$ to $11 \%$ of children suffer from sleep-disordered breathing, ranging in severity from snoring to OSA, as assessed by parent report [1]. Consequences of untreated OSA include: failure to thrive, enuresis, attention-deficit disorder, behavior problems, poor academic performance, and cardiopulmonary disease [2] [3]. The most common etiology of OSA is adenotonsillar hypertrophy.

Tonsillar surgery is the treatment of choice for most children with OSA. Moreover, OSA, rather than chronic infection, has become the primary indication for pediatric tonsillectomy, especially in younger children [4] [5]. A large recently published randomized trial supports the beneficial effects of early adenotonsillectomy, compared with a strategy of watchful waiting, for school-age children with OSA [6]. Nevertheless, pain is a common postoperative morbidity of tonsillectomy; the degree is usually severe, and often leads to poor oral intake and dehydration.

Tonsillotomy (also known as partial tonsillectomy and as intracapsular tonsillectomy) was the most commonly performed tonsillar surgery until the 1930s, when the broad introduction and safety of general anesthesia led to a preference for tonsillectomy (also known as total or traditional tonsillectomy and as subcapsular tonsillectomy) [7]. However, tonsillectomy is associated with considerable postoperative morbidity, including long recovery, characterized by dysphagia and odinophagia due to pain, bleeding, and loss of school days. Recent years have witnessed renewed interest in tonsillotomy as treatment of OSA, by means of a variety of techniques and with positive outcomes on morbidity and postoperative pain. The advantage of tonsillotomy over conventional tonsillectomy is that it leaves a residual tonsillar tissue and capsule which protects the underlying musculature with its vessels and nerves. Microdebrider partial tonsillectomy, first described by Koltai et al. in 2002 [8], and coblation were two techniques employed in tonsillotomy.

Coblation technology uses the combination of radiofrequency energy with a saline solution for gently and precisely removing tonsils, preserving the capsule, and precluding damage from surrounding healthy tissue. In prospective trials comparing the two techniques, coblation and steel cold dissection, for total tonsillectomy [9] and for adenotonsillectomy [10], fewer intraoperative and postoperative complications and less postoperative pain were observed with coblation. Numerous studies and two recent reviews [11] [12] have compared recovery-related outcomes of tonsillectomy and tonsillotomy by a variety of techniques. Less pain and more rapid recovery were reported for tonsillotomy, both by microdebrider and by coblation [11] [12]; however, since different techniques were used for tonsillectomy and tonsillotomy, it was not clear if the findings were due to the technique (microdebrider, coblation) or to the intracapsular rather than subcapsular procedure. Few studies and neither of these reviews compared the effectiveness of treating OSA between the two procedures using the same technique.

The purpose of this study was to compare recovery-related outcomes and effectiveness of tonsillectomy and tonsillotomy for the treatment of OSA in children, using the same technique, namely coblation: coblation intracapsular adenotonsillectomy (ICT) and coblation subcapsular adenotonsillectomy (CT).

\section{Material and Methods}

This is a retrospective study of children aged 2 - 18 years old who underwent coblation intracapsular adenotonsillectomy (ICT) or coblation subcapsular adenotonsillectomy (CT) for treatment of OSA at one institution, with the same surgeons, during a five year period (2007 to 2010). The indication for ICT was OSA; and for CT, OSA together with chronic tonsillitis. OSA was clinically determined in all children before surgery. Exclusion criteria were the presence of other diseases, such as cardiac, lung or metabolic and medical treatment that influences sleep.

Data retrieved from patient charts included intraoperative complications and the number of hours postoperative that the patients consumed a fluid diet. Parents were called by telephone and asked to respond to the Obstructive Sleep Apnea Syndrome Quality of Life Survey (OSA-18) [13] regarding the period before and after surgery.

The pediatric OSA-18 questionnaire is an18-item survey that comprises 5 categories (sleep disturbance, physical symptoms, emotional distress, daytime function, caregiver concerns). Questions are scored 1 (never) to 7 (persistently) based on the frequency of symptoms. The maximum score is 126 . Scores of less than 60 suggest 
a small impact on health-related quality of life (HRQL); scores between 60 and 80 suggest a moderate impact; and scores greater than 80 suggest a large impact.

In addition to the OSA-18 questionnaire, parents were asked to assess their children's pain for the 7 days postoperative as mild, moderate, or severe; their degree of satisfaction with the surgery, according to the visual analogue scale; the duration of time of recovery from tonsillectomy; postoperative complications; the use and frequency of analgesic drugs; and the time lapse, in days, until the start of solid food. Postoperative pain was also evaluated by the use of analgesic drugs and the timing of eating solid food, according to patients' charts.

The surgical procedure was performed as described previously [14], except for differences in the coblate settings of the surgical wand: 7 instead of 9 during ablation of the tonsils from the surface, and 7 instead of 6 during dissection.

Statistical analysis: The Chi-square test was used to compare data between the intracapsular and subcapsular groups.

\section{Results}

Table 1 presents demographic and clinical data according to study procedure. During the study period, 43 children, 28 boys and 15 girls, underwent ICT; and 37 children, 22 boys and 15 girls, CT. Mean OSA18 scores were 82.6 and 73.0 before surgery, in the ICT and CT groups respectively; and 25.5 and 24.6, respectively, postoperative. All the children who underwent ICT or CT with adenoidectomy were free from OSA symptoms postoperatively. Altogether, only $9 \%$ of the children were snoring post surgery, $13.5 \%$ following CT and 5\% following ICT.

No intraoperative or postoperative complications were observed in the ICT group, compared to $13.5 \%$ minor complications in the CT group, most of them dehydration $(p=0.01)$.

During the first 24 hours postoperative, $67.4 \%$ in the ICT group, and $94.6 \%$ in the CT group suffered pain, according to parents' report $(p<0.001)$. Mild pain complaints during the first 3 postoperative days were $64.5 \%$ in the ICT and $28.3 \%$ in the CT group. In the ICT group, $72 \%$ of the parents rated their children's 7 days postoperative pain as mild, compared with $21 \%$ in the CT group; $9 \%$ and $33 \%$ reported severe pain following ICT and CT respectively (Figure 1). Fewer than half (49\%) of children in the ICT group needed analgesics, compared with $73 \%$ in the CT group $(p<0.05)$. Of those who used analgesics, a higher proportion in the CT group received 3 or more doses (Figure 2).

In the ICT group 65\% ate solid food during the first 3 days post-surgery, compared to only $35 \%$ in the CT group, despite the recommendation to eat soft food during the first week. The proportion of children who only consumed fluids for 8 or more hours postoperative was lower in the ICT group (Figure 3) $(p<0.01$ ).

Table 1. Characteristics and outcomes of children who underwent coblation intracapsular adenotonsillectomy (ICT) and coblation subcapsular adenotonsillectomy (CT).

\begin{tabular}{|c|c|c|}
\hline Characteristic & ICT & $\mathrm{CT}$ \\
\hline Number of children & 43 & 37 \\
\hline Boys & 28 & 22 \\
\hline Girls & 15 & 15 \\
\hline Age (years) & range 2 - 8, median 4 & range 2 - 17, median 5 \\
\hline \multicolumn{3}{|l|}{ Mean OSA18 scores } \\
\hline Before surgery & 82.6 & 73.0 \\
\hline After surgery & 25.5 & 24.6 \\
\hline Snoring postoperative & $2(4.7 \%)$ & $5(13.5 \%)$ \\
\hline Postoperative complications & 0 & $5(13.5 \%)$ \\
\hline \multicolumn{3}{|l|}{ Reported pain } \\
\hline During 24 hours postoperative & $29(67.4 \%)$ & 35 (94.6\%) \\
\hline
\end{tabular}




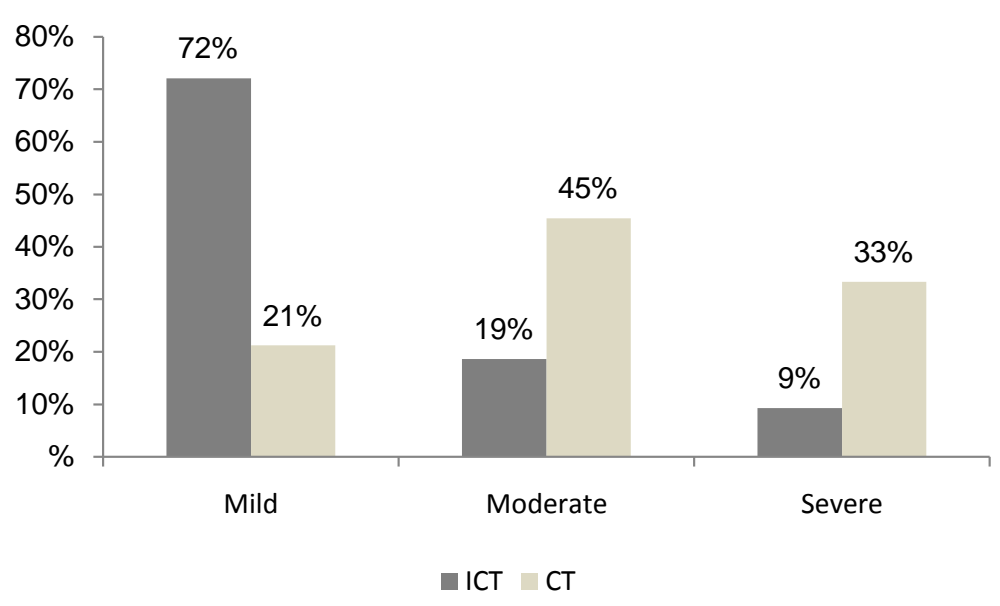

Figure 1. Pain level 7 days post surgery.

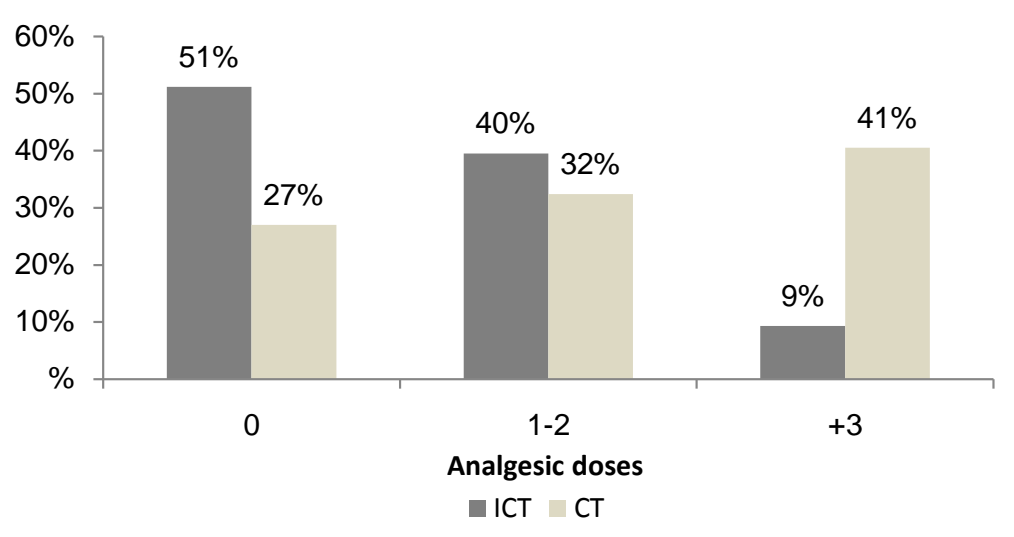

Figure 2. The number of analgesic dosages after each surgery.

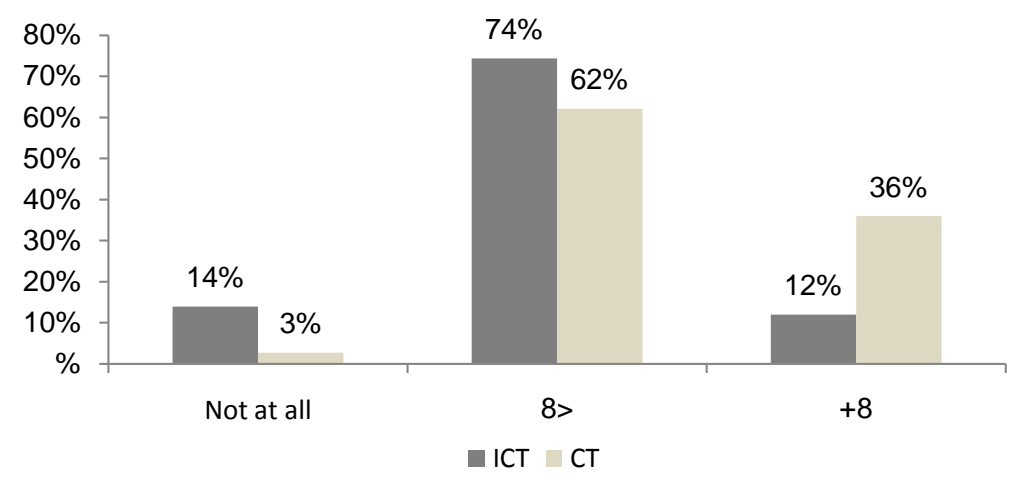

Figure 3. The number of "fluid only hours" after each surgery.

\section{Discussion}

This study compared subcapsular (total) (CT) and intracapsular adenotonsillectomy (ICT), both employing coblation. The two procedures demonstrated effectiveness as treatment for OSA, according to parent report, as well as safety, as assessed by few complications. The low thermal effect of coblation, $45^{\circ} \mathrm{C}-85^{\circ} \mathrm{C}$, reduces the risk of thermal injury to surrounding tissues, reducing pain and promoting healing.

Recovery from ICT was faster than from the subcapsular procedure, as demonstrated by fewer days with pain, less use of analgesic drugs, and earlier resumption of solid food ingestion. These findings support Acevedo et al.'s [11] subanalysis of coblation procedures, which showed a more rapid return to a regular diet and fewer 
days of analgesic use for tonsillotomy than tonsillectomy. However, that review included studies in which only tonsillotomies were performed by coblation, contrasting with the current study in which all tonsillectomies and tonsillotomies were performed using coblation.

The less pain experienced in the first 24 hours among children who underwent ICT compared to CT suggests less tissue injury in the former. Since the procedures were performed by the same two surgeons, such difference seems to be due to differences in the surgical techniques, and not to differences in the surgeons' skills. This is an important point since dissection skills of the surgeon has been suggested as a main factor determining the degree of tissue injury, and thus explains differences in the postoperative outcomes of different procedures [14].

In the current study, the lower level of pain reported by the ICT group after seven postoperative days is likely due to the protective effect of sparing the capsule. Similarly, in a randomized trial, significantly less pain was reported 5 and 6 days postoperative following intracapsular coblation tonsillectomy than following total coblation, despite comparable levels of pain between the groups on the first two postoperative days [14]. Likewise, in a prospective study in which coblation was used in a subcapsular approach on one side and intracapsular on the other side of the same patients, no difference in pain was reported within the first 24 hours [15].

Few studies have compared the effectiveness of tonsillar procedures for the treatment of OSA. The OSA-18 is a well-accepted questionnaire. Nevertheless, the use of parent report, and the time lapse from the surgery to the interviews, which was sometimes considerable, raises the possibility of reporting bias. To mitigate such bias, data were collected and correlated from patients' charts.

Despite the benefits of ICT, this procedure is not appropriate for patients suffering from tonsillitis in addition to OSA, due to the possible risk of infection in the tonsillar remnant and of regrowth of tonsillar tissue. Consequently, in the current study, CT, and not ICT, was performed in patients suffering from chronic tonsillitis. However, this raises the possibility of an indication bias, i.e. different indications for the two procedures (although all the children had OSA). In contrast, if indications were identical for the two procedures, as in randomized trials, such as those included in Walton et al.'s review [12], then some patients would evidently be over or undertreated. If, on the one hand, the indication for surgery was OSA without tonsillitis, then patients randomized to CT would be overtreated. If, on the other hand, the indication was tonsillitis, then those randomized to ICT would be undertreated. Thus, in contrast with the bias arising from the "real life" design of the current study, randomized trials comparing CT and ICT raise clinical and ethical questions.

Assessment of the effectiveness of CT for the treatment of chronic tonsillitis is beyond the scope of the current study. However, the fact that children who underwent CT suffered from chronic infection, in addition to OSA, is a limitation of this study, as well as of other such comparative studies [16]. Of note, greater postoperative bleeding was reported following operations in which recurrent tonsillitis, rather than non-infectious pathologies including sleep apnea, was the indication [17].

An explanation for the lower level of pain and complications in the ICT group is the sparing of the tonsillar capsule and the presence of remnant tonsillar tissue, which generates a protective effect that may achieve safer distance from vessels and nerves in the tonsillar bed. This mechanism is the major pathogenesis, in our opinion, for less morbidity in the ICT group and similar improvement in OSA. The benefits demonstrated herein of ICT in postoperative recovery and in relief from OSA are important, since OSA is currently the main indication for tonsillar surgery [4] [5]. Still, long term evaluation is necessary to assess the degree of tonsil regrowth following ICT, and the possible effect of such on OSA.

\section{Conclusion}

The two techniques, ICT and CT, demonstrated safety, yet there were fewer complications in the ICT group. Recovery from ICT was faster than from CT, as assessed by number of days with pain, the use of analgesic drugs and the timing to solid food ingestion. After 5 days all children in the ICT group ate solid food, while in the CT group, it took longer. In the ICT group, pain duration was shorter than in the CT group. Still, there was no significant difference in pain level between the two groups, and severe pain was not reported in either. Two thirds of the children in both groups had mild pain. The overall satisfaction from the operation and OSA symptoms was high in both groups. ICT is an effective and recommended technique for children with OSA.

\section{Acknowledgements}

The author wants to thank Ms. Cindy Cohen for her help in preparing this manuscript. 


\section{References}

[1] Lumeng, J.C. and Chervin, R.D. (2008) Epidemiology of Pediatric Obstructive Sleep Apnea. Proceedings of the American Thoracic Society, 5, 242-252. http://dx.doi.org/10.1513/pats.200708-135MG

[2] Kovacevic, L., Jurewicz, M., Dabaja, A., et al. (2013) Enuretic Children with Obstructive Sleep Apnea Syndrome: Should They See Otolaryngology First? Journal of Pediatric Urology, 9, 145-150.

[3] Marcus, C.L., Brooks, L.J., Draper, K.A., et al. (2012) American Academy of Pediatrics. Diagnosis and Management of Childhood Obstructive Sleep Apnea Syndrome. Pediatrics, 130, e714-e755. http://dx.doi.org/10.1542/peds.2012-1672

[4] Bhattacharyya, N. and Lin, H.W. (2010) Changes and Consistencies in the Epidemiology of Pediatric Adenotonsillar Surgery, 1996-2006. Otolaryngology-Head \& Neck Surgery, 143, 680-684. http://dx.doi.org/10.1016/j.otohns.2010.06.918

[5] Parker, N.P. and Walner, D.L. (2011) Trends in the Indications for Pediatric Tonsillectomy or Adenotonsillectomy. International Journal of Pediatric Otorhinolaryngology, 75, 282-285. http://dx.doi.org/10.1016/j.ijporl.2010.11.019

[6] Marcus, C.L., Moore, R.H., Rosen, C.L., et al. (2013) Childhood Adenotonsillectomy Trial (CHAT). A Randomized Trial of Adenotonsillectomy for Childhood Sleep Apnea. The New England Journal of Medicine, 368, 2366-2376. http://dx.doi.org/10.1056/NEJMoa1215881

[7] Koempel, J.A., Solares, C.A. and Koltai, P.J. (2006) The Evolution of Tonsil Surgery and Rethinking the Surgical Approach to Obstructive Sleep-Disordered Breathing in Children. Journal of Laryngology and Otology, 120, 993-1000. http://dx.doi.org/10.1017/S0022215106002544

[8] Koltai, P.J., Solares, C.A., Mascha, E.J. and Xu, M. (2002) Intracapsular Partial Tonsillectomy for Tonsillar Hypertrophy in Children. Laryngoscope, 112, 17-19. http://dx.doi.org/10.1002/lary.5541121407

[9] Omrani, M., Barati, B., Omidifar, N., Okhovvat, A.R. and Hashemi, S.A. (2012) Coblation versus Traditional Tonsillectomy: A double Blind Randomized Controlled Trial. Journal of Research in Medical Sciences, 17, 45-50.

[10] Paramasivan, V.K., Arumugam, S.V. and Kameswaran, M. (2012) Randomised Comparative Study of Adenotonsillectomy by Conventional and Coblation Method for Children with Obstructive Sleep Apnoea. International Journal of Pediatric Otorhinolaryngology, 76, 816-821. http://dx.doi.org/10.1016/j.ijporl.2012.02.049

[11] Acevedo, J.L., Shah, R.K. and Brietzke, S.E. (2012) Systematic Review of Complications of Tonsillotomy versus Tonsillectomy. Otolaryngology_Head and Neck Surgery, 146, 871-879. http://dx.doi.org/10.1177/0194599812439017

[12] Walton, J., Ebner, Y., Stewart, M.G. and April, M.M. (2012) Systematic Review of Randomized Controlled Trials Comparing Intracapsular Tonsillectomy with Total Tonsillectomy in a Pediatric Population. Archives of Otolaryngology-Head and Neck Surgery, 138, 243-249. http://dx.doi.org/10.1001/archoto.2012.16

[13] Franco Jr., R.A., Rosenfeld, R.M. and Rao, M. (2000) Quality of Life for Children with Obstructive Sleep Apnea. Otolaryngology—Head and Neck Surgery, 123, 9-16. http://dx.doi.org/10.1067/mhn.2000.105254

[14] Chang, K.W. (2008) Intracapsular versus Subcapsular Coblation Tonsillectomy. Otolaryngology-Head and Neck Surgery, 138, 153-157. http://dx.doi.org/10.1016/j.otohns.2007.11.006

[15] Arya, A.K., Donne, A. and Nigam, A. (2005) Double-Blind Randomized Controlled Study of Coblation Tonsillotomy versus Coblation Tonsillectomy on Postoperative Pain in Children. Clinical Otolaryngology, 30, 226-229. http://dx.doi.org/10.1111/j.1365-2273.2005.00970.x

[16] Cantarella, G., Viglione, S., Forti, S., Minetti, A. and Pignataro, L. (2012) Comparing Postoperative Quality of Life in Children after Microdebrider Intracapsular Tonsillotomy and Tonsillectomy. Auris Nasus Larynx, 39, 407-410. http://dx.doi.org/10.1016/j.anl.2011.10.012

[17] Khan, I., Abelardo, E., Scott, N.W., Shakeel, M., Menakaya, O., Jaramillo, M. and Mahmood, K. (2012) Coblation Tonsillectomy: Is It Inherently Bloody? European Archives of Oto-Rhino-Laryngology, 269, 579-583. http://dx.doi.org/10.1007/s00405-011-1609-8 